\title{
IMAGE SEPARATION BASED ON NONSUBSAMPLED CONTOURLET(NSCT)
}

\author{
Azadeh Bamdad Moghadam ${ }^{1}$ and Seyed Ali Asghar Beheshti Shirazi ${ }^{2}$ \\ Afshin Souzani ${ }^{3}$, Atena $\mathrm{Abdi}^{4}$ \\ ${ }^{1}$ Electrical Engineering Department, University Of Science \&Technology, Tehran, Iran \\ azadehbamdadeyahoo.com \\ ${ }^{2}$ Electrical Engineering Department, University Of Science \&Technology, Tehran, Iran \\ abeheshti@iust.ac.ir \\ ${ }^{3}$ Iran Telecommunication Research Center,Tehran,Iran \\ souzani@itrc.ac.ir \\ ${ }^{4}$ Iran Telecommunication Research Center,Tehran,Iran \\ atena_abdicaut.ac.ir
}

\begin{abstract}
When an image or a document on a paper acquired through scanning, photographing or photocopying, the image on the back page may be show through. This cause a non-linear image mixture and should be resolved through a non-linear source separation. In this paper, we improve a previously introduced method, which suppose two independent images in the same point don't have same frequency. In this work we first give a mathematical analysis for an approximate linear separation as preprocessing; then nonlinear separation procedure is improved using Non-Subsampled Contourlet Transform (NSCT) instead of normal separable wavelet transform. The NSCT provides multiscale decomposition with directional filters at each scale. Furthermore, NSCT is very efficient in saving the geometric information of images and therefore it has very good feature localization. Experimental results show that our linear preprocessing and NSCT-based non-linear separation methods both have better separation quality, comparing with previously introduce methods.
\end{abstract}

\section{KEYWORDS}

Image separation, wavelet, contourlet, non-subsampled contourlet, NSCT

\section{INTRODUCTION}

When we scan or photograph a paper document, especially if the paper is thin or transparent, the image from the back side often appears on the image from the front page. This is a routine problem which is usually occurs, when the electronic version of an old document or a book is needed.

Extracting sources from mixture images is a problem of blind source separation. Independent Component Analysis (ICA) is one of the good solutions for this problem, which is done with linear and nonlinear ICA in [1].

The results show that the nonlinear solution is better, because images are mixed through a nonlinear procedure. In [1] the nonlinear ICA, the MISEP method is used and a multilayer perceptron used for separating system. In the most of ICA methods the result are achieved from adaptive or iterative algorithms which are time consuming procedures. Another work was based on the nonlinear denoising source separation (DSS) method [3]. This method suppose that two images have independent sources and have different frequency components in the same locations. This conditions are the same as those exist when a source is contaminated by noise. 
Therefore, nonlinear denoising might be used for source separation. The separation method used here is similar to that implemented in [3]. However, authors believe that human visual perception uses different edge directions in the mixed subjects two separate them. Therefore, we improved the method in [3] using those analysis spaces that well decompose the edges in the image.

As well known, wavelet analysis offers limited directional information in representing image edges when separable one-dimensional transforms are used for images. Minh N. Do and Martin Vetterli developed a "true" two dimensional representation, called contourlet [4], which can capture the intrinsic geometrical structure that is key in visual information. Compared to the recent image representations this construction results in a flexible multiresolution, local and directional image expansion using contour segments. Moreover, subsampling process in the wavelet transform change the geometric information of image details like edges. So, nonsubsampled wavelets are introduced for decomposing the image frequency components with better saving its geometric information.For comparing the results with [5], here we use the images that that Mr. B. Luis. Almeida made in his laboratory. He dealt with a difficult version of image mixture problem, corresponding to the use of onion skin paper, which cause a strong nonlinear mixture. The source and mixture images are shown in figures 1 and 2 . More information about images is available in [5] and [6].This paper is organized as follows: Section 2 explains the basic image separation method which is used in this paper. Section 3 introduces the proposed preprocessing method. Section 4 presents separation method based on nonsubsampled contourlet transform. Section 5 explains the experimental result. Section 6 gives the concluding remarks.

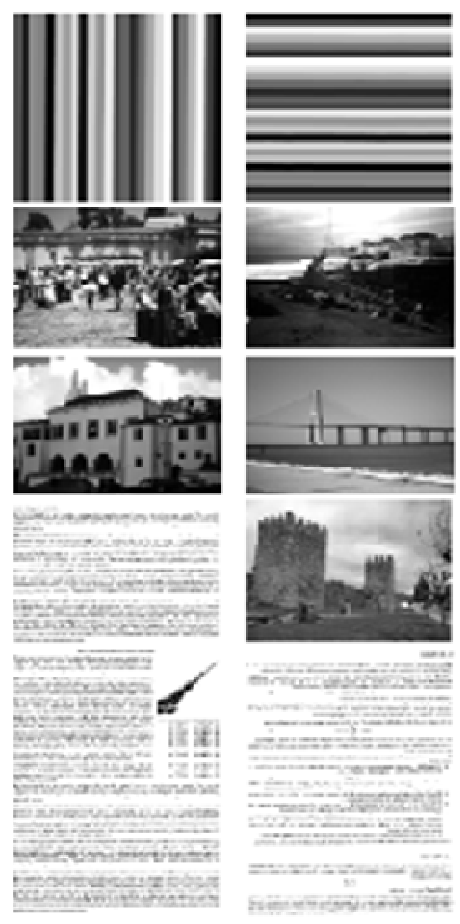

Figure 1. Source images [5] 


\section{Basic Image Separation Method}

The nonlinear image separation procedure based on denoisng source separation [1] is based on wavelet transform, and uses very basic information about the sources and the mixture procedure. This information is based on two observations:

1. The high frequency components (details) of common images are sparse. As a consequence, the wavelet coefficients from two different source images will seldom both have significant values in the same image location.

2. Each source is more strongly represented in the image acquired from that side of the paper in which that source is printed than in the image acquired from the opposite side.

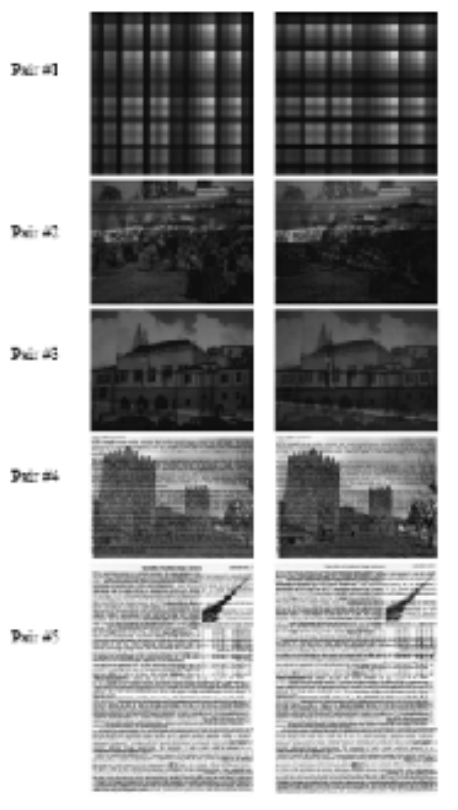

Figure 2. images after scanning [5].

The schematic representation of the separation method is shown in Figure 3. In this figure, the mixed images are first preprocessed by the following relations [5].

$$
\begin{aligned}
& p_{1}=q_{11} \cdot x_{1}+q_{12} \cdot x_{2} \\
& p_{2}=q_{21} \cdot x_{1}+q_{22} \cdot x_{2} \\
& \mathbf{Q}=\left[\begin{array}{cc}
\frac{a_{11}+a_{22}}{2} & a_{12} \\
a_{21} & \frac{a_{22}+a_{11}}{2}
\end{array}\right]
\end{aligned}
$$

Where, $\mathrm{x} 1, \mathrm{x} 2$ and $\mathrm{p} 1, \mathrm{p} 2$ are data of mixed and preprocessed image pairs, and $\mathrm{Q}=[\mathrm{qij}]$ is the Almeida's decorrelating matrix. Moreover, $A=[a i j]$ is the the square root of the autocovariance matrix of the mixture data [5]. Applying the Q matrix to the mixed image vector is a linear transformation, which is applied as a preprocessing step. Since the image mixing nature is not linear, preprocessing is followed by a nonlinear deseparating process based on wavelet analysis which iteratively decompose the approximation coefficients $\mathrm{Aj}$ (the low frequency component) at level $\mathrm{j}$ to four components (the approximation at level $\mathrm{j}+1$, and the details in three 
orientations; horizontal, vertical, and diagonal). After performing wavelet decomposition on mixed images for a certain number of levels, a competition is performed between the corresponding high frequency components from the two mixture images in each side of paper, according to following equation:

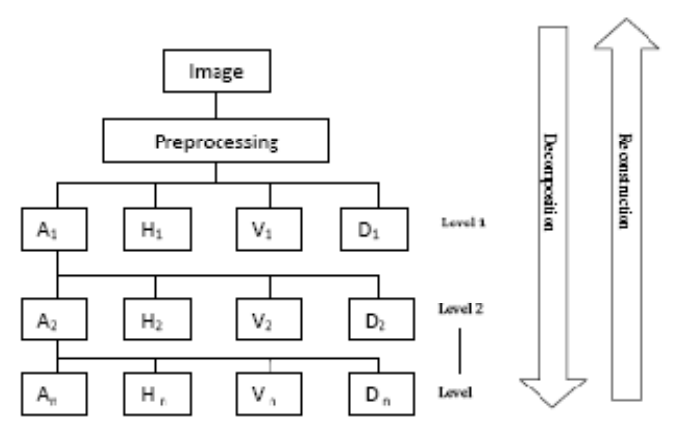

Figure 3. Schematic representation of the Wavelet-based separation method

$$
\begin{gathered}
m_{i}=\frac{1}{1+\exp \left(-a \frac{x_{i}^{2}-x_{3-i}^{2}}{x_{i}^{2}+x_{3-i}^{2}}\right)} \\
y_{i}=m_{i} \cdot x_{i}
\end{gathered}
$$

where $\mathrm{i}=\{1,2\}$ indices the two sides of the paper, xi are the wavelet coefficients of a given type (for example vertical coefficients at the first decomposition level) of the decomposition of the ith mixture image, $\mathrm{x} 3-\mathrm{i}$ are the corresponding coefficients from the other mixture image, and a is a parameter that controls the strength of the competition. This competition was applied to all horizontal, vertical and diagonal wavelet coefficients at all decomposition levels.

The competition computes mask mi through a soft winner take all function [1], and then applies this mask to the wavelet high frequency coefficients of the mixture images, so that the coefficients are intensified in the image in which they originally were stronger and weakened in the image in which they were weaker. The separated images then will be synthesized using the wavelet coefficients computed by this form of competition. In Wavelet Based Nonlinear Separation Of images [5], one-dimensional discrete wavelet basis are separately used in horizontal and vertical directions.

\section{Proposed Preprocessing Method}

In the first step we assume the mixing procedure is a linear process and try to estimate the mixing matrix and implement the inverse matrix for separation. Although, we know the linear assumption is not right at all, however, it can be used as a preprocessing step to decorrelate the mixed images.

If we show the source signals as $\mathrm{s} 1, \mathrm{~s} 2$ and mixture signals with $\mathrm{x} 1, \mathrm{x} 2$, then we have:

$$
\left[\begin{array}{l}
x_{1} \\
x_{2}
\end{array}\right]=\left[\begin{array}{ll}
a & b \\
b & a
\end{array}\right]\left[\begin{array}{l}
s_{1} \\
s_{2}
\end{array}\right]
$$


In equation (3) we assume that the variables are zero-mean. This means we deal with the mean subtracted source and mixture images.

$$
\begin{gathered}
\mathrm{E}\left\{\left[\begin{array}{l}
x_{1} \\
x_{2}
\end{array}\right]\left[\begin{array}{ll}
x_{1} & x_{2}
\end{array}\right]\right\}=\left[\begin{array}{ll}
a & b \\
b & a
\end{array}\right] \mathrm{E}\left\{\left[\begin{array}{l}
s_{1} \\
s_{2}
\end{array}\right]\left[\begin{array}{ll}
S_{1} & s_{2}
\end{array}\right]\right\}\left[\begin{array}{ll}
a & b \\
b & a
\end{array}\right] \\
{\left[\begin{array}{cc}
X_{1} & X_{12} \\
X_{12} & X_{2}
\end{array}\right]=\left[\begin{array}{ll}
a & b \\
b & a
\end{array}\right]\left[\begin{array}{cc}
S_{1} & 0 \\
0 & S_{2}
\end{array}\right]\left[\begin{array}{ll}
a & b \\
b & a
\end{array}\right]}
\end{gathered}
$$

The new parameter are defined as:

$$
\left\{\begin{array} { l } 
{ X _ { 1 } = E \{ x _ { 1 } ^ { 2 } \} } \\
{ X _ { 2 } = E \{ x _ { 2 } ^ { 2 } \} } \\
{ X _ { 1 2 } = E \{ x _ { 1 } x _ { 2 } \} }
\end{array} \quad \left\{\begin{array}{l}
S_{1}=E\left\{s_{1}^{2}\right\} \\
S_{2}=E\left\{s_{2}^{2}\right\} \\
E\left\{s_{1} s_{2}\right\}=0
\end{array}\right.\right.
$$

So, We have:

$\left\{\begin{array}{l}X_{1}=a^{2} S_{1}+b^{2} S_{2} \\ X_{2}=a^{2} S_{2}+b^{2} S_{1} \\ X_{12}=a b\left(S_{1}+S_{2}\right)\end{array}\right.$

Combining the equations in (7) and doing some manipulations we have:

$$
\frac{(a+b)^{2}}{(a-b)^{2}}=\frac{X_{1}+X_{2}+2 X_{12}}{X_{1}+X_{2}-2 X_{12}} \cong U^{2}
$$

And finally we get:

$$
\frac{b}{a}=\frac{U-1}{U+1}
$$

In other side, from the mean value of $\mathrm{x} 1$ and $\mathrm{x} 2$ we can write:

$$
\bar{x}_{1}+\bar{x}_{2}=(a+b)\left(\bar{s}_{1}+\bar{s}_{2}\right)
$$

If we assume the standard deviation has the same equation as (10), which can be almost true in images, then we can write:

$$
\sqrt{X_{1}}+\sqrt{X_{2}}=\left(\sqrt{S_{1}}+\sqrt{S_{2}}\right)(a+b)
$$

By future manipulations on equation (11) and combining the first two equation in (7), we get:

$$
\begin{gathered}
S_{1} S_{2}=\left(\frac{\sqrt{X_{1} X_{2}}}{(a+b)^{2}}-\frac{a b\left(X_{1}+X_{2}\right)}{\left(a^{2}+b^{2}\right)(a+b)^{2}}\right)^{2} \\
S_{1}+S_{2}=\frac{\left(X_{1}+X_{2}\right)}{a^{2}+b^{2}}
\end{gathered}
$$

Also we assume that $\mathrm{S} 1$ and $\mathrm{S} 2$, the variances of the source pictures are equal, then:

$$
\frac{\left(X_{1}+X_{2}\right)^{2}}{\left(a^{2}+b^{2}\right)^{2}}-4 \frac{X_{1} X_{2}}{(a+b)^{4}}-\frac{4 a^{2} b^{2}\left(X_{1}+X_{2}\right)^{2}}{\left(a^{2}+b^{2}\right)(a+b)^{4}}+\frac{8(a b) \sqrt{X_{1} X_{2}}\left(X_{1}+X_{2}\right)}{\left(a^{2}+b^{2}\right)(a+b)^{4}}=0
$$


We can calculate a and b parameters using (9) and (14) and apply the reverse matrix to mixture images:

$$
\left[\begin{array}{l}
p_{1} \\
p_{2}
\end{array}\right]=\left[\begin{array}{ll}
a & b \\
b & a
\end{array}\right]^{-1}\left[\begin{array}{l}
x_{1} \\
x_{2}
\end{array}\right]
$$

Where $\mathrm{p} 1$ and $\mathrm{p} 2$ are preprocessed images and we use them for final separation instead of $\mathrm{x} 1$ and $\mathrm{x} 2$.

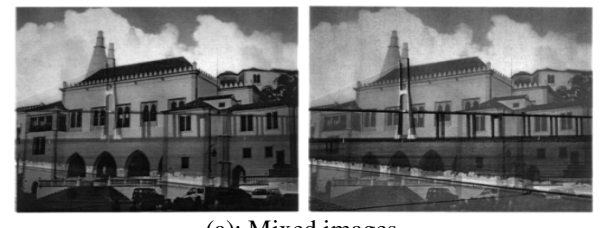

(a): Mixed images

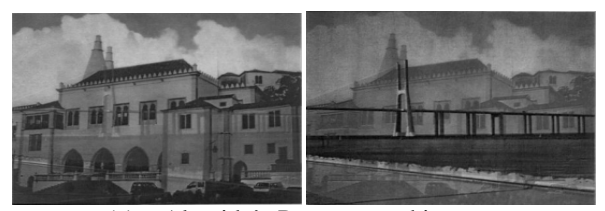

(c) : Almeida's Preprocessed images

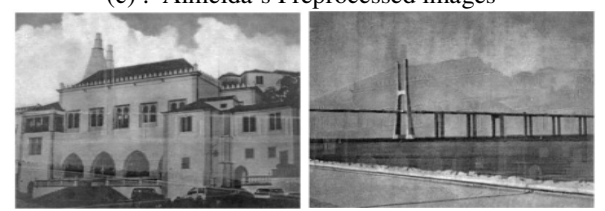

(c) : Our Preprocessed images

Figure 4. Results of implementing the proposed preprocessing method

The result of applying Q matrix of equation 1 and our estimated matrix to one pair of mixed images are shown in Fig. 4. The results show that, although both methods couldn't not completely deseparate the mixed images, the proposed method has better separation quality. This shows, our suppositions correctly estimate the mixing parameters and the proposed linear processing method is more accurate for preprocessing step.

\section{The New Nonlinear SEParation Method}

As well as [5], the nonlinear separation method used here is based on noise cancelation methods. However, we believe that human visual system separate images from two side by characterizing the direction of edges or contours in each side and supposing that the more powerful edges belongs to the front side and the weaker edges to the back side. Therefore, the most important improvement of our nonlinear separation method is to obtain sparse edges and contours. While Almeida et. al. supposed high frequency component of images are different in each side of mixed images and analyzed those by normal wavelet transform we looked for more accurate analysis methods to separate the edges in the mixed images.

Two dimensional wavelets, with tensor-product basis functions are pour in directionality and only give three direction components; i.e. horizontal, vertical and diagonal. Therefore, they can't well separate edges which have closer directions. Contourlets, however, were developed as an improvement over wavelets in terms of this inefficiency. The coutourlet transform not only has the multiscale and time-frequency-localization properties of wavelets, but also offers a high degree of directionality and anisotropy. This property of contourlet can be effectively used in our application, when we need more details in competition of components extracted in images 
scanned from each side of paper. The contourlet transform is a multi-directional and multiscale transform that is constructed by combining the Laplacian pyramid with the directional filter bank (PDFB) . The contourlet transform is shown in Figure 5; first, a multiscale decomposition into octave bands by the Laplacian pyramid is computed, and then a directional filter bank is applied to each high pass channel.

We use PDFB(Pyramid Directional Filter Bank) because it allows different number of directions at each scale while nearly achieving critical sampling as Minh N. Do and Martin Vetterliy told in their paper [4].

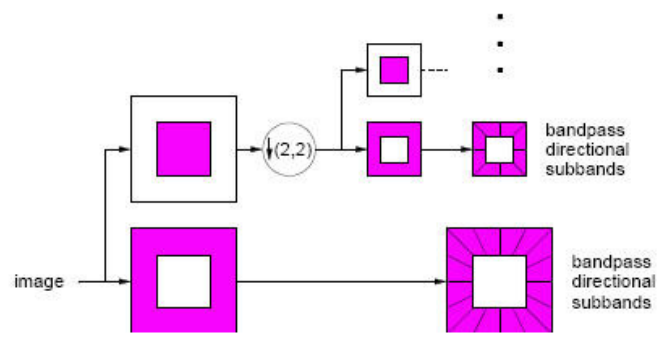

Figure 5. The frequency domain support of contourlet filter bank

In all subband or wavelet analysis schemes, It is known that downsampling makes the transform shift-variant. It means that a dyadic relation exist between the source sample and its scaled wavelet components of each level. Therefore, there is not any one by one relation between samples other than dyadic and the subsampled wavelet coefficient. However, shift-invariance is necessary for all applications that wavelet perform as an analysis tool. Specially, in image separation, when the competition is needed, the localization of each pixel is important and help us to have a better competition.

To preserve frequency partition structure of original wavelet and realize shift-invariance, the step of downsampler is removed from original filter bank [7]. Therefore, the nonsubsampled wavelet transform (NSWT) is built upon nonuniform filer banks without subsampling. In our case nonsubsampled contourlet transform (NSCT) is built upon nonsubsampled pyramids and nonsubsampled DFBs [7] and NSCT is obtained by the construction of corresponding multiscale and multi-directional filter bank. The NSCT is shift-invariant such that each pixel of the transform subbands corresponds to that of the original image in the same location. Therefore, we gather the geometric information pixel by pixel from the NSCT coefficients.

Therefore, the separation method proposed in this paper is based on a nonsubsampled contourlet which is consist of two filter banks; a pyramid structure and non-subsampled directional filter banks [7]. The result is a flexible multiscale, multidirection, and shift invariant decomposition.

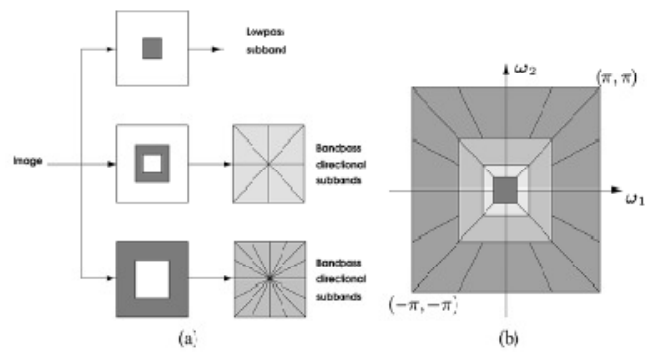

Figure 6. nonsubsampled contourlet transform. (a) nonsubsampled filter bank structure that implements the NSCT. (b) Idealized frequency partitioning obtained with the NSCT [7] 
In this paper after the decomposition by non-subsampled contourlet, we compare the high frequency content with equation (2) in section 2. The separation procedure is the same as Figure 3 , but here we have many coefficients in different directions instead of three directions of wavelet transform, also NSCT is shift invariant transform, which more accurately represents the edges and contours.

\section{EXPERIMENTAL RESULT}

The comparison of the proposed method and that of [5] preprocessing method was given in figures 4 (b) and (c). The nonlinear separation method described in sections 2 and 4 was applied to The five pairs of mixed images shown in Figure 2. Since our proposed preprocessing had better performance we used it for comparison of both methods of nonlinear processing step. The results are compared for different decomposition methods with proposed preprocessing method. The decomposition methods are: the normal wavelet transform as applied in [5] and [8] with Haar bases, the contourlet transform and the non-subsampled contourlet transform. The results of separated images with different type of decomposition are shown in Figure 7,8 and 9. The decomposition in wavelet has 7 levels for pairs 1, 2, 3; and 8 levels for pairs 4 and 5. But the level of decomposition in contourlet and NSCT was 4 for all pairs. Form figures 7,8 and 9 we can see that the result of NSCT are much better than wavelet and contourlet.

The best separation were obtained with NSCT with proposed preprocessing for all mixed pairs, also the result of using normal contourlet with proposed preprocessing is better than wavelet transform. This shows that all three proposed changes (preprocessing, contourlet and nonsubsmpled decompositions) improves the image separation.
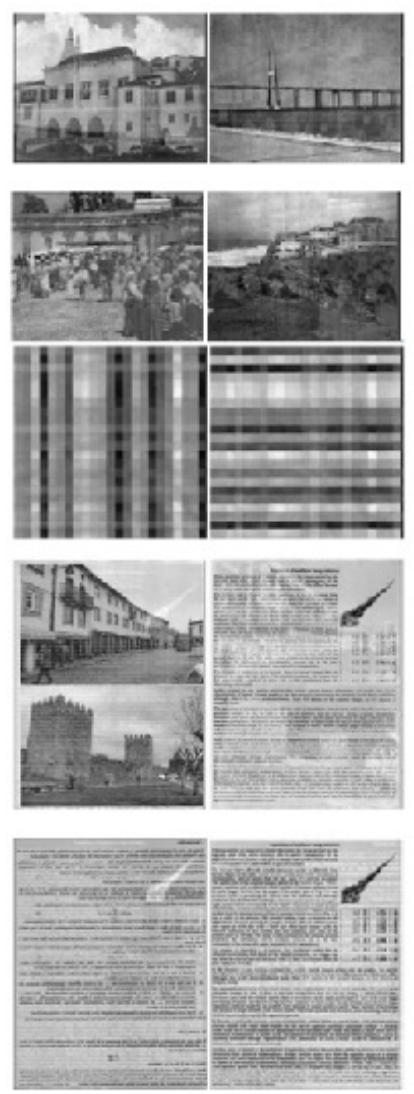
International Journal on Organic Electronics (IJOE) Vol.2, No.1, January 2013

Figure 7. The result with wavelet transform with proposed preprocessing (continued).
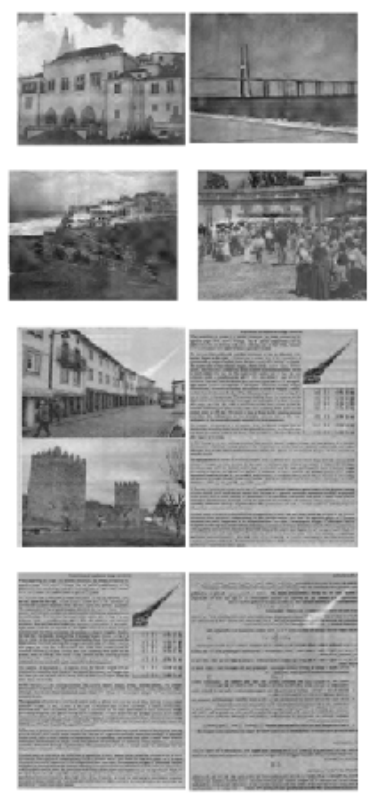

Figure 8. The results with normal contourlet transform and proposed preprocessing.
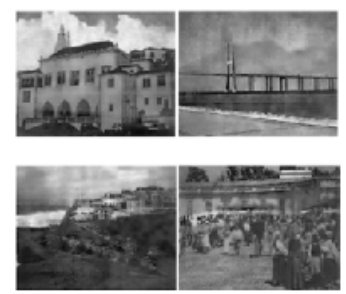

Figure 9. The results with nsct and proposed preprocessing
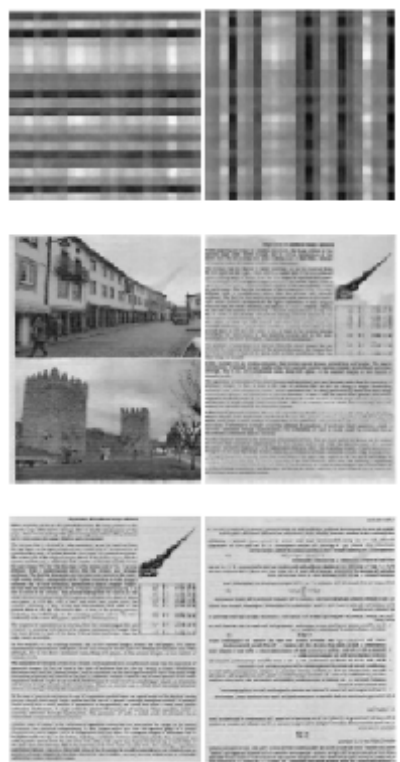

Figure 10. The results with nsct and proposed preprocessing(continued) 


\section{Conclusions}

In this paper we provided a reasonable estimation of the linear mixing parameters which results a new linear source separation method and is pretty suitable for preprocessing before final nonlinear separation. Moreover, we used NSCT, a multiscale, multidirection, and shift invariant tool for decomposition of mixed images in the nonlinear separation phase. Comparing with the normal wavelet transform, NSCT is more powerful in representing the edges and contours and subsequently improves the image separation method. Although, comparing to wavelet transform, NSCT has more calculations but very good separation results is achieved with less number of levels of decomposition. Therefore, the total separation times are almost the same as wavelet decomposition.

\section{REFERENCES}

[1] L.B. Almeida, (2003)“ MISEP-Linear and Nonlinear ICA Based On Mutual Information”, Journal of Machine Learning Research, vol. 4, pp. 1297-1318.

[2] L.B. Almeida, (2005)“Separating a Real-life Nonlinear Image Mixture”, Journal Of Machine Learning Research, vol.6, pp. 1199-1229.

[3] M.S.C.Almeida, H.Valpola and j. Sarela,(2006) "Separation Of Nonlinear Image Mixtures By Denoising Source Separation” Belin,Germany, vol. LNcs 3889, p.p.8-15, Springer-Verlag.

[4] D. Duncan, Y. Po and Minh N. Do,(2006) "Directional Multiscale Modeling of Images Using the Contourlet Transform", IEEE Transacations on image processing, vol. 15, Issue 6, pp. 1610-1620.

[5] Mariana S.C. Almeida and Luis B. Almeida,(2006) "Wavelet Based Nonlinear Separation Of images", Instituto de Telecommuincacoes, Lisboa, Portugal, 1-4244-0657-9/06.

[6] Luis.B.Almeida, http://www.lx.it.pt/ lbameida/ ica/seethrough/index.html.

[7] Arthure L.dacuha and Jianping Zhou,(2006) “The Nonsampled Transform Theory, Design and Application”, IEEE Transactins on image processing, vol.15, no.10.

[8] Mariana S.C Almeida and Luis B. Almeida,(2007)"Wavelet Based Separation of Show-through and Bleed-through Image Mixtures", Instituto de Telecommuincacoes, Lisboa, Portugal.

\section{Authors}

1. A.Bamdad Moghadam was born in marsielle in France on march 31,1979. She received her BS degree from khaje nasir toosi university of technologyin Iran,in 2002, and MS degree from Iran University of Science and Technology in 2007. She is currently a researcher in research institute for ICT in Iran . Her current research interests are Image separation, Image processing and Information security.

2. Ali Asghar Beheshti Shirazi. received the B.Sc. and M.Sc. degrees in Communication Engineering from Iran University of Science and Technology (IUST) in 1984 and 1987, respectively and Ph.D. from Okayama University, Japan in 1995. In 1995, he joined the Department of Electrical Engineering, IUST, where he currently is an Assistant Professor. His research interests include Digital Image Processing and compression, Data Communication Networking and Secure Communication.
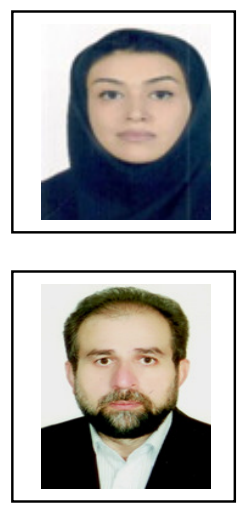